\title{
Evolutionary Ecology of Learning: Insights from Fruit Flies
}

\section{Tadeusz J. Kawecki}

Department of Ecology and Evolution, University of Lausanne, Switzerland

Email: tadeusz.kawecki@unil.ch

Population Ecology 52, 15-25

DOI 10.1007/s10144-009-0174-0

Except for a few editorial changes at the proof stage, the content of this preprint is identical with the published version.

\begin{abstract}
Ecologically and evolutionarily oriented research on learning has traditionally been carried out on vertebrates and bees. While less sophisticated than those animals, fruit flies (Drosophila) are capable of several forms of learning, and have an advantage of a short generation time, which makes them an ideal system for experimental evolution studies. This review summarizes the insights into evolutionary questions about learning gained in the last decade from evolutionary experiments on Drosophila. These experiments demonstrate that Drosophila have the genetic potential to evolve substantially improved learning performance in ecologically relevant learning tasks. In at least one set of selected populations the improved learning generalized to another task than that used to impose selection, involving a different behavior, different stimuli, and a different sensory channel for the aversive reinforcement. This improvement in learning ability was associated with reduction in other fitnessrelated traits, such as larval competitive ability and lifespan, pointing out to evolutionary trade-offs of improved learning. These trade-offs were confirmed by other evolutionary experiments where reduction in learning performance was observed as a correlated response to selection for tolerance to larval nutritional stress or for delayed aging. Such trade-offs could be one reason why fruit flies have not fully used up their evolutionary potential for learning ability. Finally, another evolutionary experiment with Drosophila provided the first direct evidence for the long-standing ideas that learning can under some circumstances accelerate and in other slow down genetically-based evolutionary change. These results demonstrate the usefulness of fruit flies as a model system to address evolutionary questions about learning.
\end{abstract}

Keywords: behavior; Drosophila; experimental evolution; learning; memory; trade-offs 


\section{Introduction}

Learning involves acquisition and storage of information. However, we cannot directly access the neural representation of past experience in the memory of an animal, nor can we ask it to tell us what it remembers. It is thus only through a change of behavior that we can find out that the animals has learned something. More importantly, any benefits of the learned information or skill for fitness can only be realized through behavior. Therefore, learning in biology is usually operationally defined as a change of an animal's behavior due to past sensory experience that the animal remembers. Defined this way, the process of learning consists of three stages: acquisition of information, its storage in memory, and, at some later time, its retrieval leading to a change in behavior.

Learning is often regarded as a form of adaptive phenotypic plasticity, but in fact learning differs from other forms of plasticity in an important way (Dukas 2004a). Plasticity means that the phenotype expressed by an individual is affected by its environment in addition to its genotype (Fig. 1a); this relationship between the phenotype and the environment is described by the reaction norm (Schlichting and Pigliucci 1998; Stearns 1992). Plasticity can become adaptive as a result of natural selection acting on genetic variation in the shape of the reaction norm, favoring responses that lead to high fitness in each environment. An example of such adaptive plasticity in behavior is the response of many birds of temperate regions to changes in photoperiod. Many of them respond to increasing day length at the end of winter by switching on behaviors anticipating breeding, such as courtship or territoriality. Conversely, decreasing day length at the end of summer induces migratory behavior in species which could not survive winter in their breeding range. These responses are obviously adaptive, as they allow the animal to anticipate the beginning of the breeding season or the coming of winter, before the breeding or winter conditions actually arrive. Such an adaptive plastic response develops by natural selection acting over generations on genetic variation in the reaction norms. Adaptive plastic responses of this type are thus specific to the environmental factors as well as to the phenotypes affected.

In contrast, learning relies on general neural mechanisms that integrate sensory input. With learning, the phenotype - the behavioral action the individual takes - not only depends on the genotype and the current environment, but also on the memory of past events, e.g., the consequences of choosing particular actions in similar circumstances in the past (Fig. 1b). Thus, in contrast to a "classic" adaptive plastic response, a learned response develops within the lifetime of an individual based on sensory feedback. This general learning machinery can be applied to new problems and challenges; it allows an animal to develop, within its lifetime, an adaptive response to a completely novel situation, never encountered in the evolutionary past of the species. For example, birds can quickly learn to press a lever or peck at particular geometric shapes to receive food, even though those shapes or levers are not encountered in the natural environment. Without learning, to develop such a response would require many generation of natural selection. In a sense, learning thus makes natural selection on behavior obsolete.

As any complex adaptation, the ability to learn is itself a product of evolution driven by natural selection. Because of this extraordinary flexibility, which can only be compared to the flexibility of vertebrate adaptive immune system, the ability to learn can be regarded as one of the top achievements of biological evolution. Furthermore, no other species is as dependent on learning and its generalization - intelligence - as ourselves. Understanding when, how, and why evolution produces improvements in learning ability would thus offer important insights into our own origin and nature.

Many decades of research on animal learning carried out by comparative psychologists have revealed that most animals are capable of some forms of learning. Concomitantly, neuroscience has identified foci of learning in the brain and has made inroads towards understanding its neuronal and molecular bases. More recent decades have seen the emergence and development of cognitive ecology, which focuses on the functions of learning in nature and on adaptive differences in learning skills between populations and species in relation to their ecology (Dukas 2004a; Healy and Braithwaite 2000; Reader and Laland 2002; Shettleworth 1999). This research program has revealed that learning plays an important role in the natural lives of many animal species, vertebrate as well as invertebrate. For example, many animals memorize landmarks to navigate in their environment (Dyer et al. 2008; Merkle and Wehner 2008; Odling-Smee et al. 2008). Some mammals and birds cache food for the winter; careful studies show that Clark's nutcrackers can memorize the locations of several thousand food caches (Kamil and Jones 1997). Predators use associative learning to develop avoidance of toxic or distasteful prey (Gittleman and Harvey 1980; Ihalainen et al. 2008), while parasitoids learn which plant volatiles lead to vulnerable host individuals (Bleeker et al. 2006). Operant learning (trial-and-error 
learning of a skill), which allows animals in captivity to learn to press a lever or peck at geometric symbols to retrieve food (e.g., Ono et al. 2002), in nature allows them to learn, e.g., how to collect nectar from novel flower species or how to handle novel prey items (Chittka and Thomson 1997). While the above examples involve animals learning from their own experience, some animals rely on learning from others (i.e., social learning). For example, most songbirds learn their song by listening to their father (Beecher and Brenowitz 2005), but birds may also learn where to find food by observing other species (Seppanen and Forsman 2007). Finally, combination of innovation with social learning, which forms the basis of our civilization, can also be observed in some other vertebrates; examples include the use of hammers of wood or stone to crack nuts by chimpanzees (Boesch et al. 1994) or food washing in Japanese macaques (Nakamichi et al. 1998). Even though the benefits of learning for fitness in natural environments have only rarely been experimentally demonstrated (but see Dukas 2004b; Dukas and Bernays 2000; Raine and Chittka 2008), they can be plausibly inferred and are intuitively well understood.

However, the benefits of learning are but one aspect of evolutionary ecology of learning; several other relevant issues have remained relatively unexplored. First, we do not know how much genetic variation for learning ability is present in natural populations; yet this variation is the raw material of evolution and so determines the potential of a species to evolve improved learning performance. Second, it is a subject of controversy to what extent evolution acts on general aspects of learning mechanisms versus on more or less independent modules specific for particular learning tasks and contexts (Bolhuis 2005; Healy and Braithwaite 2000). Third, we know little about evolutionary costs and trade-offs associated with learning, yet it is the balance of benefits and costs that determines whether natural selection favors improved learning. Finally, even though it has been proposed over 100 years ago that learning should affect genetically based evolutionary change (Baldwin 1896; Osborn 1896), this idea has until recently remained untested.

These issues are most directly addressed with evolutionary experiments, which allow one to study in real time evolutionary changes in replicate experimental populations subject to controlled selection regimes. The species which have traditionally been the focus of studies of animal learning in ecological context - mammals, birds, fish, bees - are not well suited for this approach: their generation time is long, and maintenance of large population under controlled conditions is impractical. For that reason, we have turned to the fruit flies (Drosophila melanogaster) as a study system. Fruit flies have been a favorite model system for experimental evolution studies - they are short lived (generation time can be as short as two weeks) and easy to maintain in large numbers under standard conditions. While their learning prowess falls short of that of honey or bumble bees (not to mention vertebrates), they are capable of several forms of learning, and in the last two decades they have become the favorite system to discover genes whose products are involved in the learning processes (Davis 2005). While many other small and short lived insect species might be equally suitable for evolutionary experiments on learning, using Drosophila melanogaster gives one access to the wealth of genetic information and the genetic toolkit developed for this model system. It also allows the results of evolutionary experiments to be integrated with the knowledge of the neurobiology of this species. In this paper I summarize the insights that experimental research on learning in Drosophila, much of if done in our laboratory, has offered concerning the four issues enumerated above.

\section{Genetic potential for improved learning}

If learning and intelligence are beneficial, what constrains the evolution of improved learning abilities? One could speculate that, in most species, natural selection would favor improvements in learning performance, but the response to selection is constrained by lack of relevant genetic variation. Maybe the neuronal machinery involved in learning is already so complex and fine tuned that it is difficult to improve, and so new alleles that would confer such improvements only occur very rarely. Alternatively, one might imagine that most species do harbor genetic variation that would allow them to evolve better learning abilities, but in nature this potential is not being used by evolution because the costs would be greater than the benefits. Thus, according to the first of these two hypotheses, evolution of learning would be constrained by genetics, whereas the second hypothesis accords more importance to ecology, as it is the ecology of the species that would determine the relative importance of benefits and costs of learning from the viewpoint of Darwinian fitness.

The first of these hypotheses can be addressed by imposing laboratory selection regimes on experimental populations determining to what extent their learning performance can be improved. In our laboratory we tested if a typical nature-derived population of fruit flies has the genetic potential for 
a marked improvement in learning performance in an ecologically relevant associative learning task. We subjected replicated populations to a selection regime that favored the flies' ability to associate a flavor of an oviposition substrate with an aversive bitter taste (Mery and Kawecki 2002). Every generation the flies were given a choice between two oviposition substrates, made of orange and pineapple juice. The first time they encountered these substrates (conditioning period, Fig. 2), one of them (say, pineapple) was supplemented with quinine, whose taste is aversive to flies, but which they cannot smell as it is not volatile. The flies had thus an opportunity to learn that one of the fruit odors leads to a high quality resource, while the other is associated with a bitter, unsuitable resource patch. After three hours, a new set of orange and pineapple substrates was offered, this time neither containing quinine (test period, Fig. 2). The flies that remembered which substrate had been bitter would continue to avoid this substrate even though quinine was no more present, and instead would preferentially lay their eggs on the other substrate. Their learning ability would thus be reflected in the distribution of eggs laid in the test period on the two substrates. We quantified the learning ability with a learning score, defined as the difference between the proportion of eggs laid on the orange substrate by flies previously conditioned to avoid pineapple, and the analogous proportion laid by another group of flies previously conditioned to avoid orange. The maximum learning score is thus one, and the value of zero means no learning. To impose selection for improved learning, the next generation was bred from eggs laid during the test period on the substrate that did not contain quinine (i.e., was of good quality) during the preceding conditioning period. That way flies that remembered which substrate had been good contributed more genes to the next generation. The identity (orange versus pineapple) of the "good" substrate alternated between generations (for details see Mery and Kawecki 2002). All flies were bred on a standard cornmeal medium, so they only encountered the orange and pineapple substrates in the conditioning and test period.

The base population from which the selected populations originated did not show any detectable learning in this assay, and this remained the case for unselected control populations. In contrast, within two dozen generations the populations subject to the above selection regime all evolved a robust learning response. Further assays indicated that flies from the unselected control populations are capable of some degree of learning (it would be surprising if they did not). With sufficient amount of conditioning they were able to reach similar learning scores than the selected populations, but the flies from the selected populations learned faster (Fig. 3a). They also remembered longer - they still showed a detectable preference for the substrate that had previously contained quinine after $3 \mathrm{~h}$ of "forgetting interval"; in contrast, the control populations already lost most of their response if the conditioning and test were separated by an 1-hour interval (Fig. 3b).

Thus our selected populations had the potential to evolve, within a few dozen generations, substantially improved performance in an ecologically relevant, female-specific learning task. Another evolutionary experiment (Reif et al. 2002) addressed the recognition of unreceptive females by males, which to a large degree is a learned behavior. After 21 generations, populations where the two sexes interacted over two weeks every generation became better at this male-specific learning task, compared to populations subject to a regime under which males only encountered females for 18 hours. Earlier studies showed that flies can respond to artificial selection on the avoidance of an odor previously associated with electric shock (Hewitt et al. 1983) and the extension of proboscis in response to $\mathrm{NaCl}$ preceding delivery of sugar (Lofdahl et al. 1992). However, another experiment failed to obtain a response to selection for the ability to avoid an odor previously associated with mechanical shock (Kolss and Kawecki 2008). Nonetheless, the general message of those evolutionary experiments is that Drosophila has the genetic potential to respond to selection on performance at least in some learning tasks.

\section{General learning or a specialized task?}

Does evolution act on generalized learning processes; or rather performance in different types of learning tasks depends on specialized "cognitive modules" which can evolve more or less independently? This issue is the subject of a controversy (Healy and Braithwaite 2000; Macphail and Bolhuis 2001), and the answer would contribute to understanding why our species has developed the ability to learn how to ride a bicycle, play bridge or solve differential equations. These skills could not have directly contributed to the Darwinian fitness of our Pleistocene hunter-gatherer ancestors, so they must be by-products of natural selection acting on some other aspects of learning and intelligence. 
Our selected populations have been subject to selection for improved performance in a very specific associative learning task, with orange, pineapple and bitter taste as stimuli, oviposition substrate choice as the focal behavior. Would their improved learning generalize to other situations, behaviors and stimuli? To address this question, we used another learning assay, in which the flies learn to associate an airborne odor with aversive (unpleasant) mechanical shock (Fig. 4). A group of about 50 flies is placed in a tube with airflow which can carry odors. During conditioning (Fig. 4a) the flies are first exposed for $30 \mathrm{~s}$ to odor $\mathrm{A} ; 4 \mathrm{~s}$ after they first smell the odor they are subject to the first $1 \mathrm{~s}$ bout of violent vibrations delivered by a test tube shaker; these bouts of shock are repeated every $5 \mathrm{~s}$ while odor A is presented. Thus, odor A signals imminent danger. Then the shocks cease and the odor is flushed away by clean air. Subsequently another odor B flows into the tube for $30 \mathrm{~s}$; this odor is not associated with shock. Another $60 \mathrm{~s}$ of clean air complete the conditioning cycle. Such conditioning cycles can be repeated either back-to-back (massed training) or at 15-20 min intervals (massed training). At a desired interval after the end of conditioning the flies are tested in a T-maze, in which they are given $30 \mathrm{~s}$ to choose between the two odors (Fig. 4b). If they have learnt the association between odor $A$ and the shock, they should avoid and preferentially move towards odor $B$.

The selected populations also performed substantially better in this odor-shock learning assay (Mery et al. 2007). This indicates that the aspects of learning processes that were improved in the course of our evolutionary experiment are not specific to oviposition behavior, nor have the selected files just become better about learning about pineapple and orange flavors. Rather, they have become better at associating diverse odors with aversive cues as different as bitter taste and mechanical shock, which rely on different sensory modalities. Thus, at least in this case, evolution seems to have acted on quite a general aversive learning ability.

Furthermore, with the odor-shock assay we could show that the selected populations showed improvements in the long-term memory (Mery et al. 2007). Twenty-four hours after repeated spaced conditioning, the selected flies remembered the association between an odor and the shock better than the controls. Thus, even though in the course of selection it was sufficient to remember an association between fruit flavor and bitter taste for 6 hours, the improvement extended to a substantially longer time frame.

Finally, it should be noted that the odor-shock learning assay involves a "classical" or "Pavlovian" conditioning (Rescorla 1988). The flies are exposed to the conditioned stimuli (odors) and the unconditioned stimulus (shock) with no action on their part. This is quite different from the oviposition learning task, where flies have to move towards a fruit odor, land on the substrate and taste it. During such "learning by doing", referred to as "operant" learning (Rescorla 1988), certain behavioral actions are rewarded and become strengthened, while others are punished and therefore become suppressed. In contrast, no behavior is involved in learning acquisition in the classical conditioning, i.e., there is no association between a behavior and reward or punishment. It is only during the subsequent test that the learned association between stimuli impinges upon a particular behavior. Classical and operant conditioning are therefore often regarded as involving different mechanisms (Rescorla 1988). Our experiment indicates that evolution may act on them together.

\section{Evolutionary costs of learning}

That flies can readily evolve improved learning performance in ecologically relevant tasks provokes the question, why have they not used this evolutionary potential for improved learning? One could hypothesize that the benefits of improved learning in fruit flies would not be high enough to compensate for the costs. The ability to learn is a complex adaptation which is likely to be associated with some costs or trade-offs, and so improved learning ability would be favored by natural section only if the benefits were greater than the costs. Much of the discussion focused on the costs of acquiring information during learning, i.e., the energy and time spent in the process, and the costs and risks associated with making mistakes (Dukas 2004a; Johnston 1982). However, learning ability may be associated with other costs, not directly related to the act or learning, but rather reflecting evolutionary trade-offs between learning ability and other ecologically relevant aspects of the animal's performance. One reason for such trade-offs would be the energetic cost of the central nervous system. Even though human brain only accounts for about $2 \%$ of the adult body weight, it consumes about $20 \%$ of metabolic energy at rest; in infants this share can be as high as $50 \%$ (Laughlin 2001). Thus, brain is an energetically expensive organ, and presumably being able to learn better requires a brain which is larger and more plastic in terms of synaptic connections, and so would presumably require more energy for its development, maintenance and function. Yet, each individual has only a 
limited amount of energy and other resources at disposal, and if more energy is to be invested the brain, it must be diverted from other functions such as growth, reproduction or immune defense. Evolutionary costs of learning could also reflect design trade-offs, independent of energy allocation. For example, it has been speculated that the large size of the human infant brain is a major reason for complications during childbirth, resulting in high mother and infant mortality under preindustrial conditions (Wittman and Wall 2007). It may also have been one reason why the humans evolved to be born at an earlier developmental stage than other apes.

Evolutionary experiments allow such evolutionary trade-offs associated with learning ability to be addressed directly, by studying evolutionary changes associated with the evolution of improved learning performance. With this aim, we tested if our fly populations, which had evolved improved associative learning performance, showed reductions in other fitness-related traits compared to the unselected control populations. Studying such correlated responses to selection is a direct way of addressing evolutionary trade-offs (Roff and Fairbairn 2007). We found no differences between the selected and control populations with respect to egg to adult viability, developmental time, body size or fecundity at young age under benign conditions (Burger et al. 2008). However, the "smarter" females showed a faster decline of fecundity with age and lived $15 \%$ less long than the controls (for males the difference was $10 \%$ ), indicating that they were aging faster (Burger et al. 2008). The trade-off between aging and learning worked both ways: another set of fly populations which experimentally evolved delayed aging turned out to learn poorly compared to their corresponding control populations (Burger et al. 2008). Thus, at least some of the relevant loci show antagonistic pleiotropy: alleles that improve learning reduce resistance to the effects of aging and vice versa.

Because in nature flies hardly ever live long enough to show symptoms of aging, the ecological significance of the above trade-off may be marginal. In contrast, another trade-off shown by the selected "smart" flies is likely to be of much greater importance: they suffered from reduced larval competitive ability. Because the flies from selected and control populations were indistinguishable from each other, it was not possible to study their performance in direct competition against each other - once the flies were mixed it would be impossible to tell who is who. Instead, we studied the competition of flies from each selected and control population against larvae of a strain which carried a white mutation, and so were possible to distinguish based on their white eye color. To study the competitive ability, a fixed number of eggs from a given selected or control population were placed in a vial already containing a fixed number of eggs from the white competitor strain and a very limited amount of food. This amount of food was insufficient for all the larvae to complete their development, so that less than half survived to adulthood. The proportion of red-eyed flies among the survivors was used as a measure of competitive ability of a given selected or control population against the standard white-eyed competitor. Flies from the control populations clearly performed better: they constituted about $80 \%$ survivors (only $20 \%$ being the white-eyed competitors), whereas flies from the selected populations only made up about $55 \%$ of survivors (Mery and Kawecki 2003). One could speculate that this competitive inferiority of the selected populations might be due to a higher inbreeding depression - selection results in a reduced effective population size. Analysis of crosses between replicate selected populations and between replicate control populations excluded this alternative explanation. Rather, the poor competitive performance of the selected populations seems to be due to pleiotropic effects of alleles that were favored by the learning selection regime (Mery and Kawecki 2003).

As for aging, this the trade-off between learning ability and larval development under nutritional stress also turned out to be symmetric. In another evolutionary experiment, we bred flies for the ability to develop on food which was very poor. Their adaptation to this nutritional stress involved improved viability and faster development, but, compared to corresponding controls, they showed poorer learning as adults (Kolss and Kawecki 2008).

Thus, the evolutionary experiments with Drosophila indicate that evolution of improved learning ability is associated with trade-offs with other ecologically relevant aspect of performance. These trade-offs seem to be symmetric - selection for better learning results in reduction of larval performance and faster aging, whereas selection for improved larval performance or slower aging results in reduction of learning ability. These trade-offs also seems quite specific - for example two independent evolutionary experiments indicate that learning ability does not trade-off with resistance to larval parasitoids (Kolss et al. 2006). The next challenge is to get insights into the mechanisms of those costs.

\section{How learning affects evolution}


The ability to learn is a product of evolution acting on genetic variability. But once learning has evolved in a species, it can affect the relationship between genotype and fitness, and so influence how the species evolves in response to natural selection. In particular, thanks to learning an individual may be able to compensate for inadequacies of its genetically-based phenotype with respect to the environment. If so, the ability to learn would reduce the fitness disadvantage of suboptimal genotypes and so reduce the effective strength of natural selection. In such a case learning would slow down evolutionary change (Anderson 1995; Johnston 1982; Papaj 1994). However, already at the end of the 19th century several authors proposed that under some circumstances learning may facilitate evolutionary adaptation to a novel environment (Baldwin 1896; Osborn 1896). First, because of its ability to produce adaptive changes of behavior within a lifetime, learning may allow a population to persist an environment, to which it is initially genetically maladapted, and without learning would go extinct. If the population persists in the new environment, evolution will have time to act on the genetically-based behaviors and other traits to bring them closer to the local optimum. Second, under certain circumstances, learning may accelerate evolutionary change. This scenario, known as the Baldwin effect, has been demonstrated in several specific models (Fontanari and Meir 1990; Hinton and Nowlan 1987). Paenke et al. (2007) derived more general conditions for the Baldwin effect: learning will accelerate the response to directional selection if the proportional increase of fitness brought by learning is greater in individuals which are genetically more fit. In turn Borenstein et al. (2006) showed theoretically that learning ability may allow a population to cross an "valley" in an adaptive landscape, and so more from a local optimum trait combination to another, potentially resulting in higher fitness.

Despite the long history of the concept and numerous mathematical models, until recently the Baldwin effect had not been experimentally verified. We took advantage of the learning abilities and suitability of Drosophila for experimental evolution to study the effect of learning on the response to directional selection on preference for a new resource (Mery and Kawecki 2004). As in the selection for learning ability, we used oviposition substrate choice as the focal behavior. There were four selection regimes, plus unselected control populations. In the regime Innate Orange each generation the flies were offered a choice between an orange and a pineapple substrate; the next generation was bred from the eggs laid on the orange substrate. This selection regime thus favored flies that had a stronger genetically-based preference for the orange flavor. In the regime Learning Orange the flies were also selected every generation to oviposit on the orange substrate, but they had additionally an opportunity to learn that orange should be preferred. This was done by first offering the flies for 3 hours an orange substrate and a pineapple substrate laced with quinine. Subsequently, as in regime Innate Orange the flies were given uncontaminated orange and pineapple substrates, and eggs laid at this time on orange were used to breed the next generation. Regime Learning Orange was thus identical to the regime described in Fig. 2, except that it was always pineapple that contained quinine in the conditioning period and always eggs laid on orange in the test period were used to breed the next generation. The proportion of eggs laid by a fly on the orange medium - and thus its contribution to the next generation - reflected an interaction between its innate (genetically-based) preference and the influence of its previous experience with quinine-laced (i.e., bitter tasting) pineapple. The selection regimes Innate Pineapple and Learning Pineapple were mirror images of Innate Orange and Learning Orange, respectively: the new generation was always bred from eggs laid on pineapple, and in Learning Pineapple the flies had previous experience with a quinine-laced orange substrate.

If the opportunity to learn slowed evolution, the innate preference of flies in the Learning Orange and Learning Pineapple selection regimes should change less in the course of experimental evolution than the preference of flies from the Innate Orange and Innate Pineapple regimes. In contrast, if learning facilitated evolution, the reverse should be the case. Unexpectedly, both outcomes occurred in our experiment, depending on the direction of selection. Of the populations selected to prefer orange, the Innate Orange populations evolved a stronger innate preference for orange compared to the Learning Orange populations (Fig. 5a). Thus, an opportunity to learn slowed down the evolution of geneticallybased innate preference for the orange flavor. However, for the populations selected to prefer pineapple, Learning Pineapple evolved stronger innate preference for the pineapple substrate than Innate Pineapple (Fig. 5b), indicating that for this direction of selection learning actually facilitated the genetically-based response to selection.

These seemingly contradictory results could be explained by considering the effects of learning on the relationship between genetically-based innate preference (the genotypic value as defined in quantitative genetics, Falconer and Mackay 1996) of a given individual, and its realized preference quantified as the proportion of eggs laid on the selected substrate (i.e., the phenotype), as illustrated by the following model. Consider an individual facing a choice between two resources, where resource 
$B$ is of higher quality (Fig. 6); the preference for resource $B$ can be expressed as a number between 0 (complete avoidance of $B$ ) and 1 (exclusive use of resource $B$ ). In the absence of learning, the realized phenotypic preference corresponds directly to the innate preference (assuming the availability of resource is not limiting the choice). In this case a particular difference between individuals in their innate preference $(\Delta l)$ maps linearly on a difference in their realized preference (Fig 6). How will learning change the relationship between the innate preference and the realized preference? If the individual already shows a strong innate preference for resource $B$, there is little scope for learning to increase it further. At the other end of the spectrum, if the individual shows a strong preference for the low-quality resource $A$, the effect of learning is also likely to be small, for two reasons. First, because the individual prefers resource $A$ to start with, it is not likely that it will sample resource $B$ and thus find out that resource $B$ is actually better. Second, even if it does sample resource $B$, it may not be easily convinced that $B$ is better than $A$ because this experience conflicts with its strong innate notion that $A$ should be better. This argument is supported by evidence from phytophagous insects (e.g., Potter and Held 1999), and a similar effect occurs in humans (Ohman and Dimberg 1978). In contrast, and individual with no clear innate preference for one resource over the other will likely sample both equally and will be more amenable to change its preference as a result of experience. Thus, the effects of learning on the realized resource preference should be greatest for individuals that do not show strong preference for either resource. Under this scenario, and allowing for limited amount of learning, the relationship between the innate preference and the realized preference might look like the curve in figure 6.

Now consider how learning, under this scenario, affects the difference in realized preference between two individuals whose innate preference values differ by $\Delta l$. If these individuals show moderate innate preference for resource $B$, then learning will reduce the difference in realized preference between them $(\Delta L<\Delta$ l, Fig. 6a). In contrast, if the individuals initially show preference for resource $A$, the one which is initially less biased will change its preference more, i.e., in this case learning will magnify the effect of difference in innate preference on realized preference $(\Delta L>\Delta$, Fig. 6b). The situation in figure 6a may have corresponded to the case in our experiment where orange was the high-quality resource: before the start of the experiment the mean innate preference for orange was already about 57 \% (line in Fig. 5a). Thus, learning, which could be used by flies in the Learning Orange regime, would reduce the effect of innate preference on the actual proportion of eggs laid on orange. This would reduce the effective strength of selection on innate preference (note that in our evolutionary experiment the effective contribution to the next generation was proportional to the proportion of eggs laid on the selected resource). In contrast, the initial innate preference for pineapple was only $40 \%$, which would correspond to the situation in figure $6 \mathrm{~b}$, where learning magnifies the effective selection on the innate preference.

While alternative explanations cannot be excluded (Mery and Kawecki 2004) and this model remains a hypothesis, it illustrates how in principle learning may affect the rate at which a population responds to selection.

\section{Conclusions}

The results reviewed above indicate the power of experimental evolution as the approach and Drosophila as a model system to address evolutionary questions about learning. The fruit flies are capable of learning in ecologically relevant contexts, such as resource patch choice or mating. Genetic variation in their populations allows them to evolve, in response to laboratory selection, substantially improved learning performance within as few as several dozen generations, i.e., 1-2 years. That this potential for improved learning has not been exhausted by evolution in nature can be explained by evolutionary trade-offs of learning, some of which could be identified by studying changes in fitnessrelated traits correlated with evolution of improved learning. Flies which recently evolved improved learning performance also offer an opportunity to study neuronal and genetic basis of those improvements. And, the fruit fly system permits experimental tests of hypotheses concerning the interaction between learning and evolutionary change, providing the first direct demonstration of Baldwin effect.

Even though Drosophila brain only contains about $10^{5}$ neurons (compared to about $10^{11}$ in human brain), and even though its anatomy is very different from that of vertebrate brains, the neuronal mechanisms of learning appear to be highly homologous (Davis 2005). Furthermore, all species are subject to the same forces of evolution. Thus, understanding the evolutionary and molecular aspects 
of learning in Drosophila will give us insights into the processes that have shaped the evolution of learning and intelligence in vertebrates, including us.

\section{Acknowledgements}

This work reported here was supported by the Swiss National Science Foundation, the Roche Research Foundation and the Velux Foundation.

\section{References}

Anderson RW (1995) Learning and evolution: a quantitative genetics approach. J. Theor. Biol. 175:89101

Baldwin JM (1896) A new factor in evolution. Am. Nat. 30:441-451,536-553

Beecher MD, Brenowitz EA (2005) Functional aspects of song learning in songbirds. Trends Ecol. Evol. 20:143-149

Bleeker MAK, Smid HM, Steidle JLM, Kruidhof HM, Van Loon JJA, Vet LEM (2006) Differences in memory dynamics between two closely related parasitoid wasp species. Anim. Behav. 71:1343-1350

Boesch C, Marchesi P, Marchesi N, Fruth B, Joulian F (1994) Is nut cracking in wild chimpanzees a cultural behavior. J. Hum. Evol. 26:325-338

Bolhuis JJ (2005) Function and mechanism in neuroecology: looking for clues. Animal Biology 55:457490

Borenstein E, Meilijson I, Ruppin E (2006) The effect of phenotypic plasticity on evolution in multipeaked fitness landscapes. J. Evol. Biol. 19:1555-1570

Burger JMS, Kolss M, Pont J, Kawecki TJ (2008) Learning ability and longevity: a symmetrical evolutionary trade-off in Drosophila. Evolution 62:1294-1304

Chittka L, Thomson JD (1997) Sensori-motor learning and its relevance for task specialization in bumble bees. Behav. Ecol. Sociobiol. 41:385-398

Davis RL (2005) Olfactory memory formation in Drosophila: From molecular to systems neuroscience. Annu. Rev. Neurosci. 28:275-302

Dukas R (2004a) Evolutionary biology of animal cognition. Annu. Rev. Ecol. Evol. Syst. 35:347-374

Dukas R (2004b) Male fruit flies learn to avoid interspecific courtship. Behav. Ecol. 15:695-698

Dukas R, Bernays EA (2000) Learning improves growth rate in grasshoppers. Proc. Natl. Acad. Sci. USA 97:2637-2640

Dyer AG, Rosa MGP, Reser DH (2008) Honeybees can recognise images of complex natural scenes for use as potential landmarks. J. Exp. Biol. 211:1180-1186

Falconer DS, Mackay TFC (1996) Introduction to quantitative genetics, 4th edn. Longman, Harlow

Fontanari JF, Meir R (1990) The effect of learning on the evolution of asexual populations. Complex Systems 4:401-414

Gittleman JL, Harvey PH (1980) Why are distasteful prey not cryptic? Nature 286:149-150

Healy SD, Braithwaite VA (2000) Cognitive ecology: a field of substance? Trends Ecol. Evol. 15:22-26

Hewitt JK, Fulker DW, Hewitt CA (1983) Genetic architecture of olfactory discriminative avoidance conditioning in Drosophila melanogaster. J. Comp. Psychol. 97:52-58

Hinton GE, Nowlan SJ (1987) How learning can guide evolution. Complex Systems 1:495-502

Ihalainen E, Lindstrom L, Mappes J, Puolakkainen S (2008) Can experienced birds select for Mullerian mimicry? Behav. Ecol. 19:362-368

Johnston TD (1982) Selective costs and benefits in the evolution of learning. Adv. Study Behav. 12:65-106

Kamil AC, Jones JE (1997) The seed-storing corvid Clark's nutcracker learns geometric relationships among landmarks. Nature 390:276-279

Kolss M, Kawecki TJ (2008) Reduced learning ability as a consequence of evolutionary adaptation to nutritional stress in Drosophila melanogaster. Ecol. Entomol. 33:583-588

Kolss M, Kraaijeveld AR, Mery F, Kawecki TJ (2006) No trade-off between learning ability and parasitoid resistance in Drosophila melanogaster. J. Evol. Biol. 19:1359-1363

Laughlin SB (2001) Energy as a constraint on the coding and processing of sensory information. Curr. Opin. Neurobiol. 11:475-480

Lofdahl KL, Holliday M, Hirsch J (1992) Selection for conditionability in Drosophila melanogaster. J. Comp. Psychol. 106:172-183 
Macphail EM, Bolhuis JJ (2001) The evolution of intelligence: adaptive specialization versus general process. Biol. Rev. 76:341-364

Merkle T, Wehner R (2008) Landmark guidance and vector navigation in outbound desert ants. J. Exp. Biol. 211:3370-3377

Mery F, Kawecki TJ (2002) Experimental evolution of learning ability in fruit flies. Proc. Natl. Acad. Sci. USA 99:14274-14279

Mery F, Kawecki TJ (2003) A fitness cost of learning ability in Drosophila melanogaster. Proc. R. Soc. Lond. B 270:2465-2469

Mery F, Kawecki TJ (2004) The effect of learning on experimental evolution of resource preference in Drosophila melanogaster. Evolution 58:757-767

Mery F, Pont J, Preat T, Kawecki TJ (2007) Experimental evolution of olfactory memory in Drosophila melanogaster. Physiol. Biochem. Zool. 80:399-405

Nakamichi M, Kato E, Kojima Y, Itoigawa N (1998) Carrying and washing of grass roots by freeranging Japanese macaques at Katsuyama. Folia Primatol. 69:35-40

Odling-Smee LC, Boughman JW, Braithwaite VA (2008) Sympatric species of threespine stickleback differ in their performance in a spatial learning task. Behav. Ecol. Sociobiol. 62:1935-1945

Ohman A, Dimberg U (1978) Facial expressions as conditioned stimuli for electrodermal responses: case of preparedness. Journal of Personality and Social Psychology 36:1251-1258

Ono Y, Hayashi I, Matsushima T (2002) Visual memory of shapes in quail chicks: Discrimination among 2-dimensional objects. Zool. Sci. 19:719-725

Osborn HF (1896) Ontogenic and phylogenic variation. Science 4:786-789

Paenke I, Sendhoff B, Kawecki TJ (2007) Influence of plasticity and learning on evolution under directional selection. Am. Nat. 170:E47-E58

Papaj DR (1994) Optimizing learning and its effect on evolutionary change in behavior. In: Real LA (ed) Behavioral mechanisms in evolutionary biology. Univ. of Chicago Press, Chicago, pp 133154

Potter DA, Held DW (1999) Absence of food-aversion learning by a polyphagous scarab, Popillia japonica, following intoxication by geranium, Pelargonium x hortorum. Entomol. Exp. Appl. 91:83-88

Raine NE, Chittka $L$ (2008) The correlation of learning speed and natural foraging success in bumblebees. Proceedings of the Royal Society B-Biological Sciences 275: 803-808

Reader SM, Laland KN (2002) Social intelligence, innovation, and enhanced brain size in primates. Proc. Natl. Acad. Sci. USA 99:4436-4441

Reif M, Linsenmair KE, Heisenberg M (2002) Evolutionary significance of courtship conditioning in Drosophila melanogaster. Anim. Behav. 63:143-155

Rescorla RA (1988) Behavioral studies of pavlovian conditioning. Annu. Rev. Neurosci. 11:329-352

Roff DA, Fairbairn DJ (2007) The evolution of trade-offs: where are we? J. Evol. Biol. 20:433-447

Schlichting CD, Pigliucci M (1998) Phenotypic evolution. A reaction norm perspective. Sinauer, Sunderland

Seppanen JT, Forsman JT (2007) Interspecific social learning: Novel preference can be acquired from a competing species. Current Biology 17:1248-1252

Shettleworth SJ (1999) Cognition, evolution, and behavior. Oxford Univ. Press, Oxford

Stearns SC (1992) The evolution of life histories. Oxford Univ. Press, Oxford

Wittman AB, Wall LL (2007) The evolutionary origins of obstructed labor: Bipedalism, encephalization, and the human obstetric dilemma. Obstet. Gynecol. Surv. 62:739-748 
(a) Phenotypic plasticity

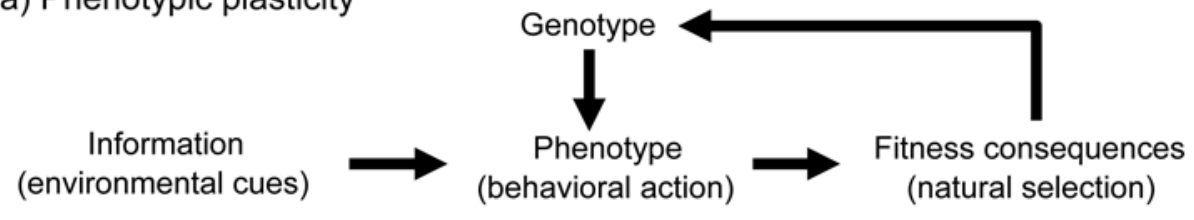

(b) Learning

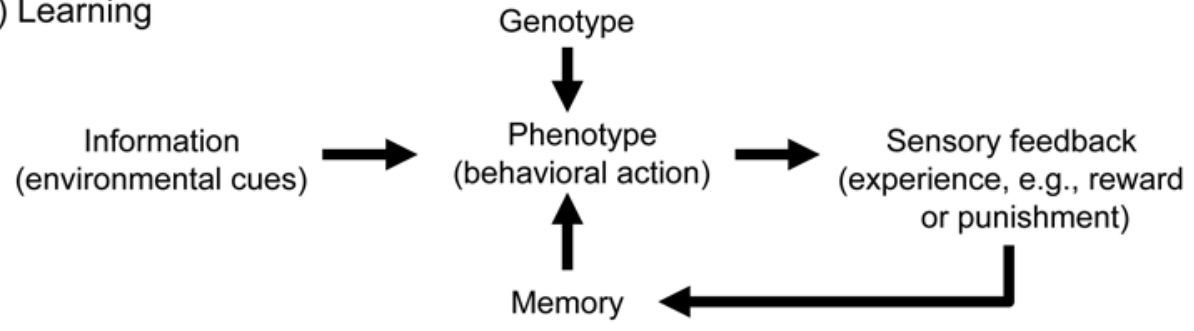

Figure 1. Schematic comparison between adaptive phenotypic plasticity of behavior (a) and learning (b). The feedback that makes plasticity adaptive is provided by natural selection acting over generations at the level of the population. In contrast, adaptive learning responses develop within a lifetime of an individual based on the feedback provided by sensory information.

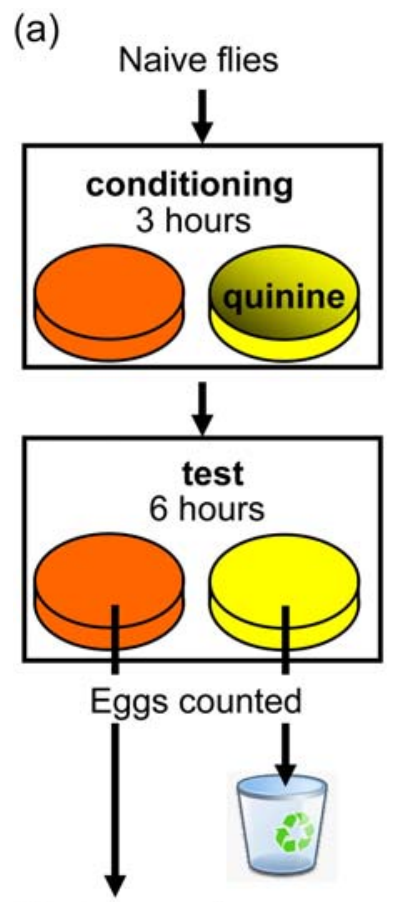

Next generation (b)
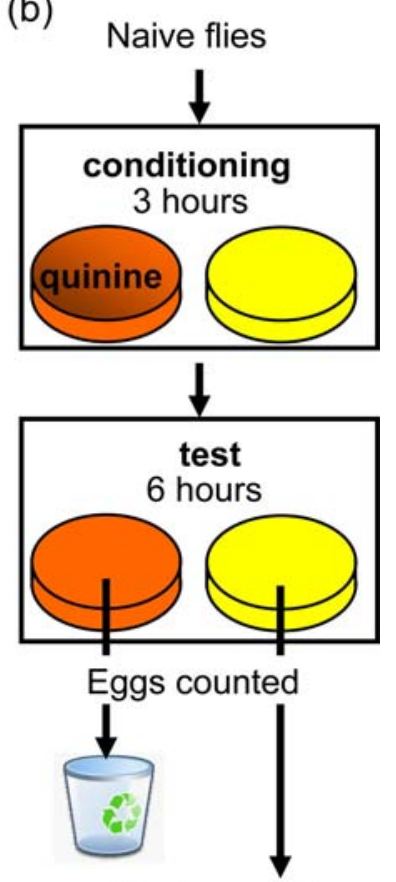

Next generation

Figure 2. The oviposition learning assay. During the conditioning period the flies have the opportunity to associate the flavor of the substrate (orange or pineapple) with the bitter taste of quinine; the other substrate does not contain quinine. During the test period the two substrates are again offered, but quinine is no more present. The difference in the proportion of eggs laid on the orange substrate by flies conditioned with quinine + pineapple (a) versus flies conditioned with quinine + orange $(b)$ is used as a learning score. To impose selection for learning, the next generation is exclusively bred from eggs laid in the test period on the substrate that did not contain quinine in the conditioning period. Modified from Mery and Kawecki (2002). 

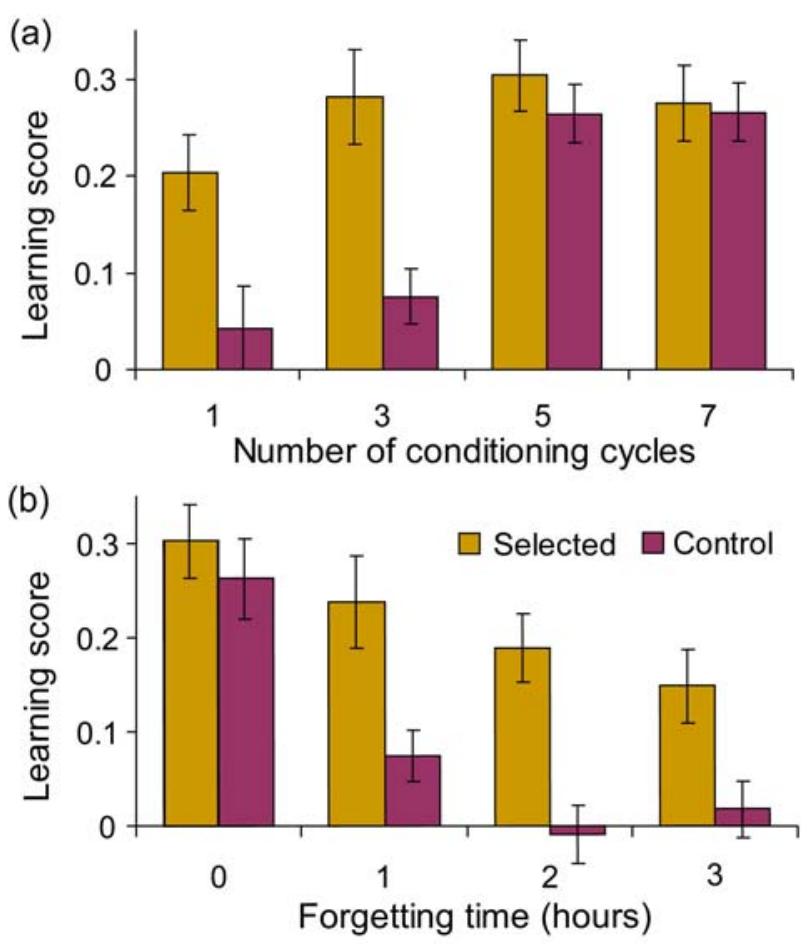

Figure 3. Experimental evolution of improved learning ability. Learning performance (means $\pm S E$ ) of seven populations selected over 40 generations for improved learning ability (yellow/light shaded) and six unselected control populations (purple/dark shaded). (a) Learning acquisition curve: learning scores as a function of the amount of conditioning (number of conditioning cycles), test immediately following conditioning. (b) Memory decay: learning scores depending on the time between the end of conditioning and the beginning of the test period (forgetting time); all flies were subject to five conditioning cycles. Learning was assayed using a modified oviposition learning assay, for details see Mery and Kawecki (2002). Data from Mery and Kawecki (2002).

(a) Conditioning

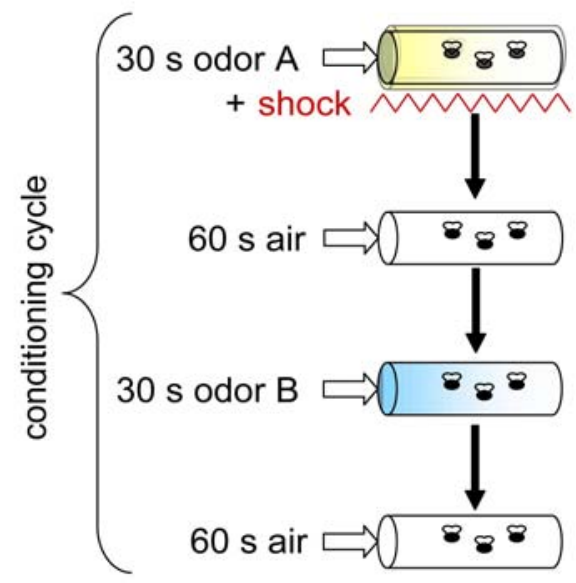

(b) Test

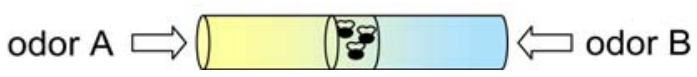

Figure 4. Schematic representation of the odor - mechanical shock learning assay. (a) Conditioning. (b) Test. For details see text. 
(a)

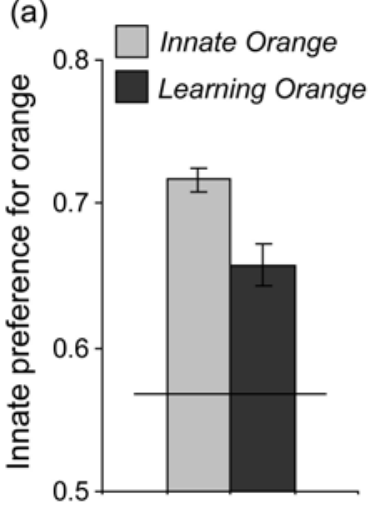

(b)

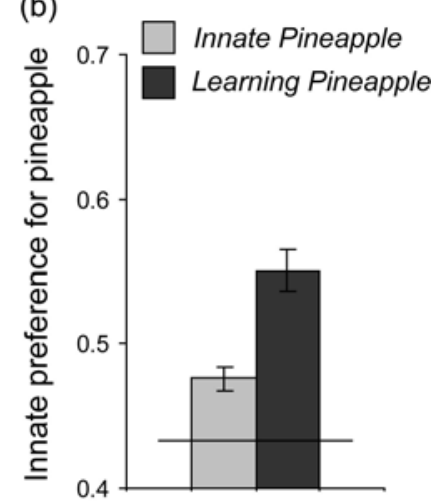

Figure 5. The effect of learning on experimental evolution or innate resource preference. (a) Populations selected to prefer orange. (b) Populations selected to prefer pineapple. Innate preference scored as the proportion of eggs laid on the focal medium by naïve flies, measured after 23 generations of selection. Bars are means \pm standard errors based on variation among replicate selection populations (eight per selection regime). The line indicates the initial innate preference of the base population (i.e., the baseline). Note that in in panel (a) it is the preference for orange and in panel (b) the preference for pineapple, so the baseline in (b) equals 1 - baseline in (a). For details see the text; data from Mery and Kawecki (2004).
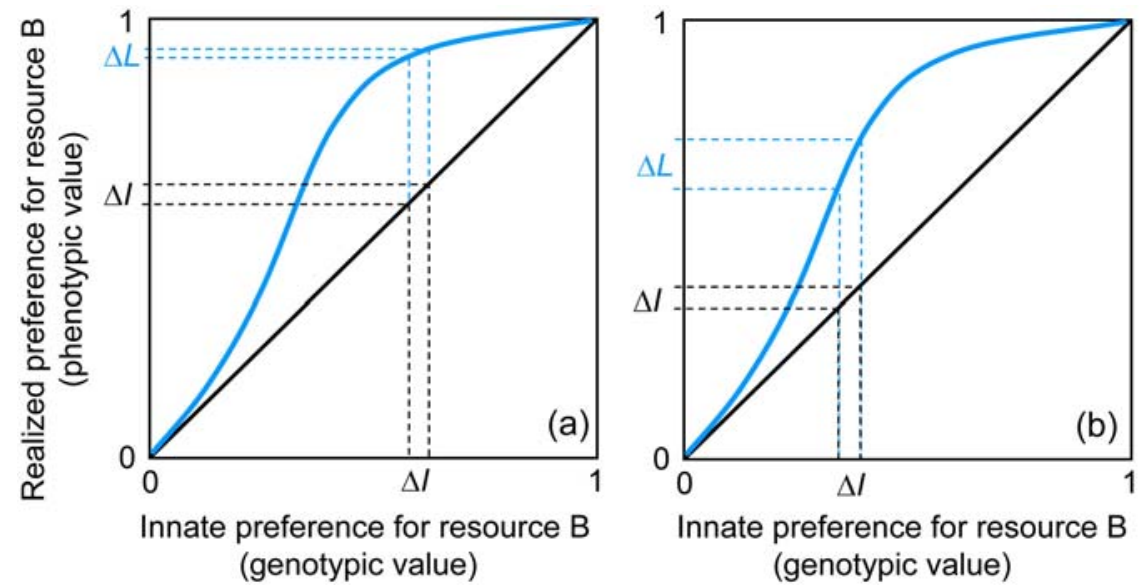

Figure 6. Graphical model of a hypothetical relationship between the genotypic value for and the realized phenotypic preference. In the absence of learning, the genotypic preference maps linearly onto the realized preference (diagonal line). When the animals show some learning, the relationship follows the curve (for justification see the text). Depending on the range of innate preferences, learning may either reduce (a) or magnify (b) the effect of a small difference in innate preference on the realized preference. $\Delta l$ : difference in innate preference; $\Delta L$ : difference in realized preference as modified by learning. 